\title{
La descripción teórica de la electropolimerización de compuestos hidroquinónicos, obtenidos electroquímicamente
}

\author{
DOI: $10.46932 / \mathrm{sfjdv} 2 \mathrm{n} 2-111$
}

Received in: March 1st, 2021

Accepted in: May 30th, 2021

\section{Volodymyr V. Tkach}

Universidad Nacional de Chernivtsi, 58000, Calle de Kotsyubynsky. 2, Chernivtsi, Ucrania Universidade Federal de Mato Grosso do Sul, Av. Sen. Felinto. Müller, 1555, C/P. 549, 79074-460, Campo Grande, MS, Brasil

\section{Marta V. Kushnir}

Universidad Nacional de Chernivtsi, 58000, Calle de Kotsyubynsky. 2, Chernivtsi, Ucrania

\section{Olga V. Skrypska}

Universidad Nacional de Chernivtsi, 58000, Calle de Kotsyubynsky. 2, Chernivtsi, Ucrania

\section{Yana G. Ivanushko}

Universidad Estatal de Medicina de Bucovina, 58001, Pl. Teatral, 9, Chernivtsi, Ucrania

\section{Sílvio C. De Oliveira}

Universidade Federal de Mato Grosso do Sul, Av. Sen. Felinto. Müller, 1555, C/P. 549, 79074-460, Campo Grande, MS, Brasil

\section{Petro I. Yagodynets}

Universidad Nacional de Chernivtsi, 58000, Calle de Kotsyubynsky. 2, Chernivtsi, Ucrania

\section{Olga V. Luganska}

${ }^{4}$ Universidad Nacional de Zaporizhzhia, 69600, Calle de Zhukovsky, 66, Zaporizhzhia, Ucrania

\section{Zholt O. Kormosh}

Universidad Nacional del Este Europeo, 43000, Avda. de la Libertad., 13, Lutsk, Ucrania Correo-e: nightwatcher2401@ gmail.com

\section{RESUMEN}

En este trabajo, por la primera vez se ha descrito el proceso de polimerización electroquímica de los compuestos hidroquinónicos, obtenidos electroquímicamente. El análisis del modelo matemático correspondiente mediante la teoría de estabilidad lineal y análisis de bifurcaciones ha mostrado que el comportamiento de este sistema se hace bastante semejante al ya observado durante la "paradoja de politiofeno". Así, hay una mayor probabilidad del acaecer las inestabilidades oscilatoria y monotónica. El polímero, por su vez, se obtendrá con una conductividad reducida y morfología menos uniforme.

Palabras-clave: compuestos hidroquinónicos, polímeros conductores, electropolimerización, la paradoja de politiofeno, estado estacionario estable. 


\section{INTRODUCCIÓN}

Las hidroquinonas [1 - 6] forman uno de los grupos de compuestos aromáticos, abundantes en fuentes naturales. Su uso incluye no solo sistemas bioaceptables, sino los sintéticos. Por ejemplo, compuestos como dopamina, catecol, ácido gálico, ácido elágico se usan tanto en formulaciones farmaceuticas, como en la síntesis de polímeros conductores [7 - 10]. La presencia de hidroxilo fenólico añade la biocompatibilidad de los polímeros y su capacidad mediadora de tranferencia de electrones.

Otrosí, los compuestos hidroquinónicos y afines son un objeto bastante popular en procesos electroanalíticos [11 - 15]. Una amplia variedad de modificadores de electrodo se usa para asistir su detección electroanalítica, a comenzar desde los polímeros conductores [16 - 19], óxidos e hidróxidos de metales [20 - 22], líquidos iónicos [23 - 24] y hasta modificadores extraordinarios como cáscara de plátano [25 - 26] o nuez de coco [27 - 28]. En el trabajo [29] la forma hidroquinónica se hace presente tanto en el modificador (polímero del colorante rojo de alizarina) como en el propio analito (omeprazol en su forma parcialmente oxidada). Las hidroquinonas se pueden obtener química y electroquímicamente. Ambas las vías se suelen usar para su polimerización.

Sin embargo, las transformaciones electroquímicas de los compuestos orgánicos (incluyendo su electropolimerización) suelen acompañarse por las inestabilidades electroquímicas (especialmente, la oscilatoria y monotónica) [30 - 32]. Estas inestabilidades pueden influenciar fuertemente la morfología del polímero resultante, llevando a deformaciones serias, responsables por valores de conductividad del polímero menores de lo previsto.

Para prever la ocurrencia de las inestabilidades mencionadas, así como las condiciones del mejor desempeño del proceso electrosintético, es necesario el análisis teórico a priori del comportamiento del sistema.

Por eso, el objetivo general de este trabajo es una investigación mecanística matemática del sistema con la electropolimerización de monómeros hidroquinónicos, obtenidos electroquímicamente. Para su realización, nosotros resolvemos objetivos específicos como:

- Sugerir un mecanismo como secuencia de reacciones químicas y electroquímicas, que llevan a la deposición de un nuevo material;

- Desarrollar el modelo matemático, capaz de describir de manera adecuada el comportamiento del sistema, según el mecanismo desarrollado en la etapa anterior;

- Del análisis del modelo derivar las condiciones de estabilidad de estado estacionario y de las principales inestabilidades (oscilatoria y monotónica); 
- Comparar el comportamiento del sistema con él de los semejantes [33 - 35].

\section{EL SISTEMA Y SU MODELO}

En la primera etapa, el electrodo, cubierto por una capa de benceno o fenol monoatómico se oxida, a potencial alto, rindiendo una hidroquinona (1):

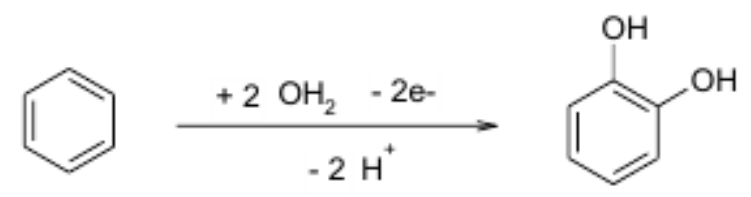

(1)

Después, el proceso de la oxidación del monómero y del polímero se realiza por el mecanismo híbrido (Fig. 1)

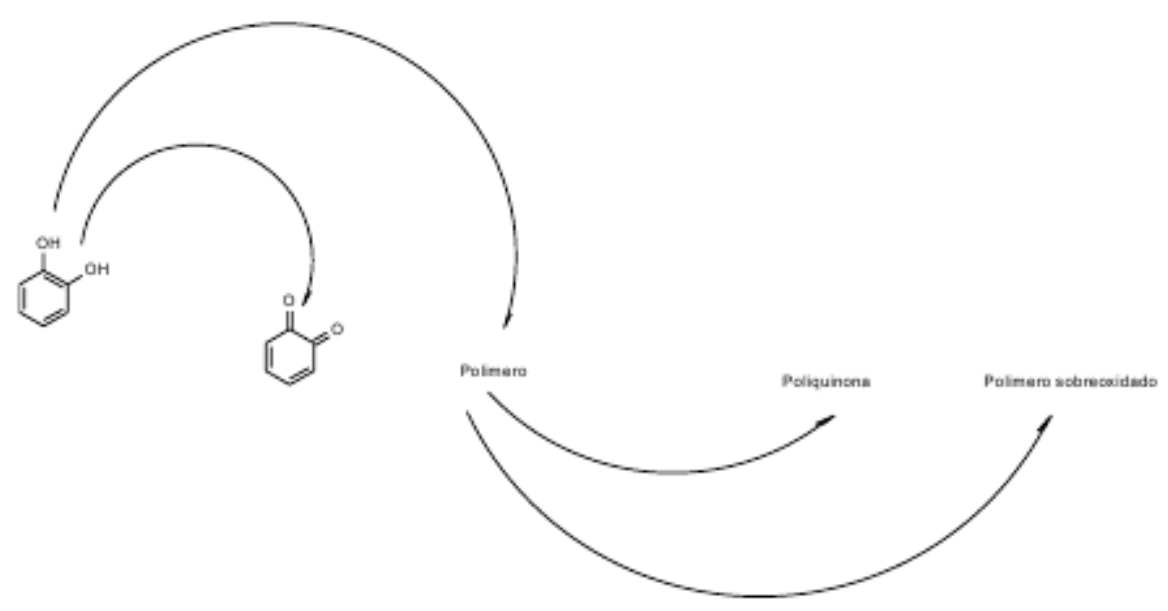

Fig. 1. El esquema del proceso electrooxidativo de la hidroquinona

Así, para describir el comportamiento del sistema, nosotros introducimos tres variables: $\mathrm{m}$ - el grado de recubrimiento del ánodo por el monómero hidroquinónico, obtenido por la reacción (1);

$\mathrm{q}$ - el grado de recubrimiento del ánodo por el compuesto quinónico, el producto de oxidación de baja molecularidad;

$\mathrm{p}$ - el grado de recubrimiento del ánodo por el polímero en su forma reducida. 
Se puede mostrar que, supuesta la cobertura total del ánodo por el compuesto inicial (benceno) en el inicio del proceso, su comportamiento será descrito por el conjunto de ecuaciones diferenciales (2):

$$
\left\{\begin{array}{c}
\frac{d m}{d t}=\frac{1}{M}\left(r_{1}-r_{q}-r_{p}\right) \\
\frac{d q}{d t}=\frac{1}{Q}\left(r_{q}-r_{p}\right) \\
\frac{d p}{d t}=\frac{1}{P}\left(r_{p}-r_{p q}-r_{S}\right)
\end{array}\right.
$$

Siendo M, Q y P las concentraciones superficiales máximas del monómero, de la quinona y del polímero y los parámetros $r$ velocidades de respectivas reacciones, que se calculan conforme $(3-7)$ :

$$
\begin{aligned}
& r_{1}=k_{1}(1-m-q-p) \exp \frac{2 F \varphi_{0}}{R T} \\
& r_{q}=k_{q} m \exp \frac{2 F \varphi_{0}}{R T} \\
& r_{p}=k_{p} m^{x} q^{y} \exp \frac{z F \varphi_{0}}{R T} \\
& r_{p q}=k_{p q} p \exp \frac{2 s F \varphi_{0}}{R T} \\
& r_{S}=k_{S} p \exp \frac{h F \varphi_{0}}{R T}
\end{aligned}
$$

Aquí, los parámetros k son las constantes de velocidades de respectivas reacciones, $\mathrm{x}$ e y son los órdenes de reacciones del monómero y su quinona en la electropolimerización, z es el número de electronos transferidos durante la electropolimerización, s es el número de unidades en la cadena polimérica, h es el número de electronos transferidos durante la sobreoxidación, $\mathrm{F}$ es el número de Faraday, $\varphi_{0}$ es el salto del potencial, relativo al de carga cero, $\mathrm{R}$ es la constante universal de gases y $\mathrm{T}$ la temperatura absoluta del vaso.

Como se ve, se trata de un proceso puramente electroquímico, no acompañado por etapas químicas. Este proceso tendrá un impacto grande sobre la doble capa eléctrica (DCE), lo que le hará asaz dinámico, según se expondrá abajo. 


\section{RESULTADOS Y DISCUSIÓN}

Para investigar el comportamiento del sistema con la electrosíntesis de poli(hidroquinonas), obtenidas electroquímicamente, acompañada por la oxidación del polímero sintetizado, analizamos el conjunto de ecuaciones diferenciales (2), así como las relaciones algébricas (3-7) mediante la teoría de estabilidad lineal. Los elementos estacionarios de la matriz funcional de Jacobi pueden ser calculados conforme (8):

$$
\left(\begin{array}{lll}
a_{11} & a_{12} & a_{13} \\
a_{21} & a_{22} & a_{23} \\
a_{31} & a_{32} & a_{33}
\end{array}\right)
$$

Siendo:

$$
\begin{gathered}
a_{11}=\frac{1}{M}\left(-k_{1} \exp \frac{2 F \varphi_{0}}{R T}+j k_{1}(1-m-q-p) \exp \frac{2 F \varphi_{0}}{R T}-k_{q} \exp \frac{2 F \varphi_{0}}{R T}-\right. \\
\left.j k_{q} m \exp \frac{2 F \varphi_{0}}{R T}-x k_{p} m^{x-1} q^{y} \exp \frac{z F \varphi_{0}}{R T}-j k_{p} m^{x} q^{y} \exp \frac{z F \varphi_{0}}{R T}\right) \\
a_{12}=\frac{1}{M}\left(-k_{1} \exp \frac{2 F \varphi_{0}}{R T}+d k_{1}(1-m-q-p) \exp \frac{2 F \varphi_{0}}{R T}-d k_{q} m \exp \frac{2 F \varphi_{0}}{R T}-\right. \\
\left.y k_{p} m^{x} q^{y-1} \exp \frac{z F \varphi_{0}}{R T}-d k_{p} m^{x} q^{y} \exp \frac{z F \varphi_{0}}{R T}\right)(10) \\
a_{13}=\frac{1}{M}\left(-k_{1} \exp \frac{2 F \varphi_{0}}{R T}+f k_{1}(1-m-q-p) \exp \frac{2 F \varphi_{0}}{R T}-f k_{q} m \exp \frac{2 F \varphi_{0}}{R T}-\right. \\
\left.f k_{p} m^{x} q^{y} \exp \frac{z F \varphi_{0}}{R T}\right)(11) \\
a_{21}=\frac{1}{Q}\left(k_{q} \exp \frac{2 F \varphi_{0}}{R T}+j k_{q} m \exp \frac{2 F \varphi_{0}}{R T}-x k_{p} m^{x-1} q^{y} \exp \frac{z F \varphi_{0}}{R T}-j k_{p} m^{x} q^{y} \exp \frac{z F \varphi_{0}}{R T}\right)
\end{gathered}
$$

$$
\begin{aligned}
& a_{22}=\frac{1}{Q}\left(d k_{q} m \exp \frac{2 F \varphi_{0}}{R T}-y k_{p} m^{x} q^{y-1} \exp \frac{z F \varphi_{0}}{R T}-d k_{p} m^{x} q^{y} \exp \frac{z F \varphi_{0}}{R T}\right) \\
& a_{23}=\frac{1}{Q}\left(f k_{q} m \exp \frac{2 F \varphi_{0}}{R T}-f k_{p} m^{x} q^{y} \exp \frac{z F \varphi_{0}}{R T}\right)(14) \\
& a_{31}=\frac{1}{P}\left(x k_{p} m^{x-1} q^{y} \exp \frac{z F \varphi_{0}}{R T}+j k_{p} m^{x} q^{y} \exp \frac{z F \varphi_{0}}{R T}-j k_{p q} p \exp \frac{2 s F \varphi_{0}}{R T}-\right.
\end{aligned}
$$

$\left.j k_{S} p \exp \frac{h F \varphi_{0}}{R T}\right)$

$$
a_{32}=\frac{1}{P}\left(y k_{p} m^{x} q^{y-1} \exp \frac{z F \varphi_{0}}{R T}+d k_{p} m^{x} q^{y} \exp \frac{z F \varphi_{0}}{R T}-d k_{p q} p \exp \frac{2 s F \varphi_{0}}{R T}-\right.
$$

$\left.d k_{S} p \exp \frac{h F \varphi_{0}}{R T}\right)$ 


$$
a_{33}=\frac{1}{P}\left(f k_{p} m^{x} q^{y} \exp \frac{z F \varphi_{0}}{R T}-k_{p q} \exp \frac{2 s F \varphi_{0}}{R T}-f k_{p q} p \exp \frac{2 s F \varphi_{0}}{R T}-k_{S} \exp \frac{h F \varphi_{0}}{R T}-\right.
$$

$\left.f k_{S} p \exp \frac{h F \varphi_{0}}{R T}\right)$

Observando los elementos de la diagonal principal de la matriz Jacobiana, se puede ver que existen elementos, capaces de ser positivos. Estos elementos son responsables por la positiva conexión de retorno (es decir, comportamiento autocatalítico o afín) y, por consiguiente, por el comportamiento oscilatorio.

Como el proceso de la electropolimerización se acompaña por cambios cíclicos de conductividades de superficie, resistencia y capacitancia de la superficie del ánodo, así como de la DCE, el comportamiento oscilatorio en este caso se hará más probable que en sistemas semejantes [33 - 35]. Matemáticamente se expresa por la positividad de los elementos $j k_{1}(1-m-q-p) \exp \frac{2 F \varphi_{0}}{R T}, \quad-j k_{q} m \exp \frac{2 F \varphi_{0}}{R T}$, $-j k_{p} m^{x} q^{y} \exp \frac{z F \varphi_{0}}{R T}, d k_{q} m \exp \frac{2 F \varphi_{0}}{R T},-d k_{p} m^{x} q^{y} \exp \frac{z F \varphi_{0}}{R T}, f k_{p} m^{x} q^{y} \exp \frac{z F \varphi_{0}}{R T},-f k_{p q} p \exp \frac{2 s F \varphi_{0}}{R T} \mathrm{y}$ $-f k_{S} p \exp \frac{h F \varphi_{0}}{R T}$. Como todos estos elementos son relacionados a los parámetros de la DCE, fuertemente dependientes de su composición, la amplitud y la frecuencia de las oscilaciones también se hará dependiente de la composición del electrólito de soporte (incluyendo el pH).

Para investigar la estabilidad del estado estacionario, nosotros aplicamos al conjunto de ecuaciones diferenciales (2) el criterio de estabilidad Routh-Hurwitz. Para simplificar el análisis del determinante de la matriz, introducimos nuevas variables, de modo que se reescriba el determinante como (18):

$$
\frac{1}{M Q P}\left|\begin{array}{ccc}
-\Lambda-\Sigma-K & -\Sigma-\Phi-\Xi & -\Omega-\Gamma-\Delta \\
\Sigma-K & \Phi-\Xi & \Gamma-\Delta \\
K-T & \Xi-\Pi & \Delta-P
\end{array}\right|
$$

Abriendo los paréntesis y aplicando el requisito Det $\mathrm{J}<0$, saliente del criterio, nosotros obtenemos la condición de estabilidad, expuesta como (19):

$$
\begin{gathered}
-(\Lambda+\Sigma+K)(\Phi \Delta+2 \Xi \Delta+\Phi P+\Xi P-\Xi \Gamma+\Pi \Gamma-\Pi \Delta)-(\Sigma+\Phi+\Xi)(\Gamma K-\Gamma T+\Delta T- \\
\Delta \Sigma+P \Sigma-P K)-(\Omega+\Gamma+\Delta)(\Sigma \Xi-\Sigma \Pi+K \Pi-K \Phi+T \Phi-T \Xi)<0 \quad(19)
\end{gathered}
$$

Correspondiente a un sistema electrosintético asaz dinámico, correspondiente a uno de los casos de la "paradoja de politiofeno", en la que las influencias desestabilizadoras de la secuencia de reacciones electroquímicas hace aún más estrecha la zona topológica de estabilidad de estado estacionario. 
Eso se manifiesta fuertemente en las propiedades morfológicas (menos uniforme) y eléctricas (menos conductor). Sin embargo, este polímero tendrá capacidad para implementar funciones de

- Capa protectora de corrosión;

- Modificador de electrodo para la detección electroanalítica de varias sustancias.

La naturaleza (total o parcialmente) quinónica del polímero le da afinidad a sustancias que, al oxidarse, transfieren un número par de electrones. Otra ventaja del polímero será su capacidad de recibir protones, mediando, así, un proceso electroanalítico de una amplia variedad de compuestos.

Otrosí, la presencia de grupos de oxígeno en el polímero sobreoxidado da posibilidad de reaccionar con metales transitivos formando complejos. Como el polímero complejo generalmente tiene mayor conductividad de corriente que el polímero sobreoxidado, la presencia de los metales transitivos se permitirá saber por el trecho creciente en la curva de conductividad.

Cuando el estado estacionario está en vigor, el determinante tiene valores negativos. Ya cuando el determinante se anula, se obtiene el límite de detección, descrito por la inestabilidad monotónica. Su punto sirve de margen entre estados estacionarios estables e inestables y, para el caso, se describe como:

$$
\begin{gathered}
-(\Lambda+\Sigma+K)(\Phi \Delta+2 \Xi \Delta+\Phi P+\Xi P-\Xi \Gamma+\Pi \Gamma-\Pi \Delta)-(\Sigma+\Phi+\Xi)(\Gamma K-\Gamma T+\Delta T- \\
\Delta \Sigma+P \Sigma-P K)-(\Omega+\Gamma+\Delta)(\Sigma \Xi-\Sigma \Pi+K \Pi-K \Phi+T \Phi-T \Xi)=0 \quad(20)
\end{gathered}
$$

En algunos casos, el compuesto quinónico no participa en la polimerización, por cuestiones estéricas o porque disuelve y desaparece del medio de la reacción (superficie). Así, la quinona se hará indiferente, y el modelo (2) será simplificado, porque la variable q ya no podrá pertenecer al conjunto de ecuaciones diferenciales. Este se transformará en (21):

$$
\left\{\begin{array}{l}
\frac{d m}{d t}=\frac{1}{M}\left(r_{1}-r_{q}-r_{p}\right) \\
\frac{d p}{d t}=\frac{1}{P}\left(r_{p}-r_{p q}-r_{S}\right)
\end{array}\right.
$$

El análisis del modelo (21) solo vendrá a confirmar lo expuesto en el modelo (2).

\section{CONCLUSIONES}

El análisis de estabilidad del sistema con la síntesis electroquímica de poli(hidroquinonas) a partir de monómeros obtenidos electroquímicamente dejó concluir que el comportamiento 
oscilatorio se realiza con mayor probabilidad, por cuenta de las influencias cíclicas de las etapas electroquímicas a la doble capa. El polímero se hará menos uniforme y menos conductor, pero con mayor aptitud al uso como capa protectora y modificador para análisis electroquímico de metales transitivos y sustancias que se oxidan dando un número par de electrones. 


\section{REFERENCIAS}

1. E. A. Haidar, Sh. A. Tawfik, C. Stampfl et al., Adv. Theor. Simuk., 4(2021), 202000203

2. N.A. Al-Shar'I, Q.A. Al-Balas, M.A. Hassan et al., Acta Pharm., 71(2021), 115

3. Y. Li, L. Mei, X. Guan et al., Food Res. Int., 142(2021), 110184

4. S. Yoganathan, A. Alagaratnam, N. Acharekar, J. Kong, Cells, 10(2021), 458

5. A. Bagheri, S. Ebrahimpour, N. Nourbakhsh et al., Arch. Oral Biol., 125(2021), 105088

6. S. Mazzotta, G. Carullo, F. Sciubba et al., Molbank, 2021(2021), M1203

7. Y Zamudio-Cuevas, M.A. Andonegui-Elguera, A. Aparicio-Juárez et al., Inflammation, 44(2021), 174

8. N. Sabbaghi, M. Noroozifar, K. Kerman, C, 7(2021), 43

9. I. Singh, G. Dhawan, S. Gupta et al., Front. Microbiol., 11(2021), 607099

10. Y. Fu, J. Zhang, Y. Wang et al., Carbohydr. Polym., 257(2021), 117598

11. L.C. Almeida, T. Frade, R.D. Correia et al., Sci. Rep. 11(2021), 2237

12. H.L.C. Maganto, M.B. M. Yap Ang, G. V. C. Dizon et al., Membranes, 11(2021), 216

13. N.A.Ertas, E. Kavak, F. Salman et al., Electroanal., 32(2020), 1178

14. J. Xue, Ch. Yao, N. Li et al., J. Electroanal. Chem., 886(2021), 115133

15. B. Bai, C. Gu, X. Lu et al., Nano Res., 137(2021), 3376

16. L. Sasso, A. Heiskanen, F. Diazzi et al., Analyst, 138(2013), 3651

17. X. Bai, B. Zhang, X. Hu et al., Bioelectrochem., 132(2020), 107398

18. S.J. Park, J. Lee, S.E. Seo et al., Sci. Rep., 10(2020), 3772

19. B. Demirkan, S. Bozkurt, K. Cellat et al., Sci Rep., 10(2020), 2946

20. J.S. Bonini, F.Q. Mariani, E. Guimarães Castro et. al., OrbitalElec. J. Chem., 7(2015), 318

21. Y. Kumar, V. K. Vashistha, D. K. Das et al., Lett. Appl. NanoBioSci.,, 9(2020), 866

22. A.C. de Sá, S. C. Barbosa, P.A. Raymundo-Pereira et al., Chemosensors, 8(2020), 103

23. L. Scarpetta, A. Mariño, K. Bolaños et al., Rev. Colomb. Cien. Quím. Farm., 44(2015), 215 
24. L.H. de Oliveira, A.C. Dias Souza, L. Pizzuti, et. al., Orbital. Elec. J. Chem., 6(2014), 255

25. J. B. Raoof, A. Kiani, R. Ojani, R. Valliolahi, Anal. Bioanal. Electrochem., 3(2011), 59

26. Zs. Öri, A. Kiss, A.A. Ciucu et al., Sens. Act. B. Chem., 190(2014), 149

27. X. Wang, D. Gao, M. Li et al., Sci. Rep., 7(2017), 7044

28. E. P. Shuaib, P. Muhammed Shafi, G. K. Yogesh et al., Mat. Res. Express, 6(2019), 115610

29. K.R. Mahantesha, B.E. Kumara Swamy, K. Vasantakumar Pai, Anal. Bioanal. Electrochem., 6(2014), 234

30. I.Das, N.R.Agrawal, S.A.Ansari, S.K.Gupta, Ind. J. Chem, 47A(2008), 1798

31. I.Das, N. Goel, S. K. Gupta, N.R. Agrawal, J. Electroanal. Chem, 670(2012), 1

32. M.S. Ba-Shammakh. Thes. Ph. D. King Fahd University of Petroleum and Minerals, Dharan, Saudi Arabia, 2002

33. V. Tkach, M.V. Kushnir, S.C. de Oliveira et al., Ukr. Bioorg. Acta, 15(2020), 47

34. V.V. Tkach, B. Kumara Swamy, R. Ojani et. al., Rev. Colomb. Cien. Quím. Farm., 44(2015), 148

35. V.V. Tkach, V.V. Nechyporuk, P. I. Yagodynets, Rev.Colomb. Cien. Quím. Farm., 41(2012), 203 